https://helda.helsinki.fi

\title{
Testing brains with burst suppressions
}

\section{Vanhatalo, Sampsa}

2016-08

Vanhatalo, S 2016 , ' Testing brains with burst suppressions ', Clinical Neurophysiology , vol. 127 , no. 8 , pp. 2919-2920 . https://doi.org/10.1016/j.clinph.2016.04.018

http://hdl.handle.net/10138/224474

https://doi.org/10.1016/j.clinph.2016.04.018

publishedVersion

Downloaded from Helda, University of Helsinki institutional repository.

This is an electronic reprint of the original article.

This reprint may differ from the original in pagination and typographic detail.

Please cite the original version. 
Editorial

\section{Testing brains with burst suppressions}

See Article, pages 2921-2930

Distinguishing burst suppression (BS) pattern from a "continuous EEG" pattern is perhaps the first and easiest to learn by junior fellows as they start working in units with neurological intensive care (Westhall et al., 2015). Detection of BS is considered the hallmark of severe compromise in brain function, and in the context of brain damage, it is traditionally considered to signify a poor prognosis (Westhall et al., 2016; Hofmeijer et al., 2014). Recognition of BS is important for neurologists when treating status epilepticus using anesthesia that is titrated to keep the EEG at the "BS level", between the levels of continuous EEG and inactive EEG.

Traditionally, recognition of BS used to be based on observing a constant background pattern that does not change over time or does not react to patient handling, and it is hence considered "unreactive". Long term EEG monitoring of patients with acute brain injury has changed the clinical perception of BS, which is now viewed as a brain state that usually changes over time. For instance, prognosis after acute asphyxia (van Rooij et al., 2005) or cardiac arrest (Oh et al., 2015) can be done from counting the hours from the initial insult to the evolution of EEG from the BS pattern to recovery of continuous EEG pattern (comprehensively reviewed by Hofmeijer and van Putten, 2016). Conceivably, such dynamics in brain states also imply that BS is not a discrete, unitary state, but the functional brain status can vary substantially within a broader category of BS states. Recent clinical studies have, indeed, provided computational evidence for quantifiable dynamics in BS that may predict clinical outcomes (Iyer et al., 2014; van Putten et al., 2015; Hofmeijer et al., 2014).

A general feature in neurological assessments is "reactivity" to exogenous stimuli, which can be testing of various reflexes, or response to handling and commands. In comatose patients, these observations are combined in an assessment scale, such as the commonly used Glascow Coma Scale (GCS). However, these scales are not very sensitive in discriminating between classes of patients in deeper comatose states, such as those with BS pattern in their EEG.

The study of Nita et al. (2016) in this issue of Clinical Neurophysiology takes an important step in this direction. They show that BS pattern in the EEG after traumatic brain injury may react to exogenous stimulations, which is delivered here using the conventional photic stimulation (PS) available in all standard EEG devices. In their work, Nita et al. delivers PS at $1 \mathrm{~Hz}$ for a period of one minute, and they find that the global field power of EEG is significantly increased during PS as compared to the immediately preceding baseline period. This "BS reactivity" was found in all five pediatric patients with different etiologies of brain trauma, and the level of their "BS reactivity" depended on the baseline BS, as well as the clinical level of patient's coma. For a neurophysiologist, it was especially interesting that PS did not affect the heart rate which was used to exclude the possibility of a more general arousal. Hence, the EEG-recorded BS reactivity likely discloses a global feature of cortical brain function that cannot be approached using other clinical measures in these patients.

Several prior studies have examined BS reactivity in deeply anesthetized humans as well as in experimental animal models (referenced in Nita et al.). While those earlier findings are compatible with the observations by Nita et al., it is important to recognize that they don't directly compare with the clinically relevant group of traumatic BS more than at the level of EEG phenomenology. The work of Nita et al. studying BS in genuinely brain injured patients is opening a novel pathway for future clinical studies. It shows that recognition of BS pattern in the EEG is just the start, and the cortical function can be readily probed deeper by using simple methods that are already available at bedside.

Future studies will be warranted to address some key properties of BS reactivity on its way to more standardized clinical practice. First, larger patient populations with more diverse etiologies and with more different age groups will be needed. The aim here should be to both establish the general utility of BS reactivity, as well as to establish quantitative norms for different ages and etiologies. Second, it would be interesting to learn whether BS reactivity is global, as shown in the study of Nita and colleagues. One could also expect that different sensory modalities (PS, auditory, tactile) might trigger region specific responses, which by themselves have further information value in differential diagnostics in the severely brain damaged patients. Third, the evolution of BS reactivity over time will need further exploration, akin to the already employed monitoring of cortical reactivity using conventional evoked potential paradigms (Fisher et al., 2016; Koenig and Kaplan, 2015). Finally, the method of computing BS reactivity by Nita et al. was relatively simple, though appropriated for a proof of concept study. The future studies can take the computational challenge to the next level and seek to define more adaptive, interactive, and even automated (Liberman et al., 2013) ways to quantify BS reactivity to make its estimate directly available for the bedside practitioner. 


\section{Acknowledgements}

The work of Dr. Vanhatalo has been supported by Academy of Finland (\#276523 and \#288220).

Conflict of interest: None to declare.

\section{References}

Fisher J, Huang S, Ye M, Nabili M, Wilent W, Krauthamer V, et al. Real-time detection and monitoring of acute brain injury utilizing evoked electroencephalographic potentials. IEEE Trans Neural Syst Rehabil Eng 2016 Mar 1. http://dx.doi.org/10.1109/TNSRE.2016.2529663.

Hofmeijer J, Tjepkema-Cloostermans MC, van Putten MJ. Burst-suppression with identical bursts: a distinct EEG pattern with poor outcome in postanoxic coma. Clin Neurophysiol 2014;125:947-54.

Hofmeijer J, van Putten MJ. EEG in postanoxic coma: prognostic and diagnostic value. Clin Neurophysiol 2016;127:2047-55.

Iyer KK, Roberts JA, Metsaranta M, Finnigan S, Breakspear M, Vanhatalo S. Novel features of early burst suppression predict outcome after birth asphyxia. Ann Clin Transl Neurol 2014;1:209-14.

Koenig MA, Kaplan PW. Clinical applications for EPs in the ICU. J Clin Neurophysiol 2015;32:472-80.

Liberman MY, Ching S, Chemali J, Brown EN. A closed-loop anesthetic delivery system for real-time control of burst suppression. J Neural Eng 2013;10:046004.

Nita DA, Moldovan M, Sharma R, Avramescu S, Otsubo H, Hahn CD. Burstsuppression is reactive to photic stimulation in comatose children with acquired brain injury. Clin Neurophysiol 2016;127:2921-30.
Oh SH, Park KN, Shon YM, Kim YM, Kim HJ, Youn CS, et al. Continuous amplitudeintegrated electroencephalographic monitoring is a useful prognostic tool for hypothermia-treated cardiac arrest patients. Circulation 2015;132:1094-103.

van Putten MJ, Tjepkema-Cloostermans MC, Hofmeijer J. Infraslow EEG activity modulates cortical excitability in postanoxic encephalopathy. J Neurophysiol 2015;113:3256-67.

van Rooij LG, Toet MC, Osredkar D, van Huffelen AC, Groenendaal F, de Vries LS Recovery of amplitude integrated electroencephalographic background patterns within 24 hours of perinatal asphyxia. Arch Dis Child Fetal Neonatal Ed 2005;90:F245-51.

Westhall E, Rosén I, Rossetti AO, van Rootselaar AF, Wesenberg Kjaer T, Friberg H, et al. Interrater variability of EEG interpretation in comatose cardiac arrest patients. Clin Neurophysiol 2015;126:2397-404.

Westhall E, Rossetti AO, van Rootselaar AF, Wesenberg Kjaer T, Horn J, Ullén S, et al. Standardized EEG interpretation accurately predicts prognosis after cardiac arrest. Neurology 2016 Feb 10. pii: 10.1212/WNL.0000000000002462.

Sampsa Vanhatalo

Department of Clinical Neurophysiology, HUS Medical Imaging, University of Helsinki and Helsinki University Hospital, Finland

URL: http://www.babacenter.fi

Available online 30 April 2016 